\title{
Synthesis of Thinned Planar Antenna Array Using Multiobjective Normal Mutated Binary Cat Swarm Optimization
}

\author{
Lakshman Pappula and Debalina Ghosh \\ School of Electrical Sciences, Indian Institute of Technology Bhubaneswar, Odisha 751013, India \\ Correspondence should be addressed to Lakshman Pappula; lp10@iitbbs.ac.in
}

Received 19 October 2015; Accepted 23 March 2016

Academic Editor: Christian W. Dawson

Copyright ( 2016 L. Pappula and D. Ghosh. This is an open access article distributed under the Creative Commons Attribution License, which permits unrestricted use, distribution, and reproduction in any medium, provided the original work is properly cited.

\begin{abstract}
The process of thinned antenna array synthesis involves the optimization of a number of mutually conflicting parameters, such as peak sidelobe level, first null beam width, and number of active elements. This necessitates the development of a multiobjective optimization approach which will provide the best compromised solution based on the application at hand. In this paper, a novel multiobjective normal mutated binary cat swarm optimization (MO-NMBCSO) is developed and proposed for the synthesis of thinned planar antenna arrays. Through this method, a high degree of flexibility is introduced to the realm of thinned array design. A Pareto-optimal front containing all the probable designs is obtained in this process. Targeted solutions may be chosen from the Pareto front to satisfy the different requirements demonstrating the superiority of the proposed approach over multiobjective binary particle swarm optimization method (MO-BPSO). A comparative study is carried out to quantify the performance of the two algorithms using two performance metrics.
\end{abstract}

\section{Introduction}

Antenna arrays are widely used in several applications including radar, satellite communications, remote sensing, biomedical imaging, and ground communications. Many of these applications call for the use of wide aperture antenna arrays. Wide aperture antenna arrays contain large number of antenna elements and they are difficult to implement due to high cost and increased mechanical complexity. Also it is difficult to control the shape of the desired radiation pattern without disturbing the uniform feeding network. In such cases aperiodic arrays obtained by altering the positions of the antenna elements may provide great flexibility in controlling the sidelobe levels while maintaining uniform amplitude distribution.

An aperiodic antenna array may be obtained by modifying a periodic antenna array in different ways. One common approach is to change the positions of the elements of a periodic array to produce aperiodic spacing between them. But in practice, shifting the positions of the antenna elements is a difficult and complex process, especially when the number of array elements is large. The alternative is to use the method of thinning [1-12]. It means turning off some elements in an equally spaced array while maintaining the radiation properties of the original array. Thinned arrays have practical advantages such as reduction in weight, cost, power consumption, and lower complexity of the feed network. The synthesis of thinned antenna arrays is a challenging problem and many thinning techniques have been proposed over the last 5 decades. As the array gets more populated, it becomes highly complex to choose the optimized array aperture from among the large number of possible solutions. Although various analytical methods have been suggested for thinned array synthesis, it becomes impractical to use such methods for large array synthesis. On the other hand, the use of optimization techniques has led to significant improvements in thinned array design, due to their global search capability and their ability to handle large number of variables in highly nonlinear scenarios. Evolutionary algorithms such as genetic algorithm (GA) [2-5], simulated 
annealing [6], particle swarm optimization (PSO) [7], ant colony optimization (ACO) [8], and differential evolution (DE) [9] have been successfully applied in thinned array synthesis.

Till date emphasis has been given to the application of evolutionary algorithms for finding optimal solutions of single objective array synthesis problems. But, in practice aperiodic antenna array synthesis is multiobjective in nature requiring the simultaneous optimization of multiple conflicting parameters. For example, the strategic elimination of antenna elements suppresses the peak sidelobe level (PSLL), but it has a detrimental effect on the shape of the main beam measured in terms of the first null beam width (FNBW). Thus, it is to be noted that the requirements of low PSLL, narrow FNBW, and minimum number of active elements are in contrast to each other. Thus, the performance cannot be improved significantly for one aspect without degrading the performance of the other. Depending on the application, it may become imperative to sacrifice gain and beam width in order to achieve lower PSLL. To achieve the best compromised solution, it is necessary to obtain a set of solutions whereby none of the solutions in the set are dominated by any other solution. This set of nondominated solutions forms the Pareto front. In recent years, several multiobjective optimization techniques [11-22] have been applied for unequally spaced linear, planar, and circular array synthesis to determine the Pareto front. This idea is carried forward in this communication for minimization of the above-mentioned conflict parameters to form the Pareto front.

This paper addresses the synthesis of planar thinned antenna arrays using MO-NMBCSO method, marking the first application of the MO-NMBCSO method for solving antenna array synthesis problems. Cat swarm optimization (CSO) is inspired from the natural behavior of cats and introduced by Chu and Tsai in 2007 [23]. It is a powerful optimization computational tool for searching continuous valued spaces. It has been applied to different engineering real valued problems $[24,25]$ and has shown better performance over well-known algorithms. The optimization for thinned array synthesis has to be formulated as a discrete variable optimization with the solutions encoded as binary strings. Thus, for thinned array synthesis a discrete optimization algorithm needs to be developed. So in this paper, a novel optimization algorithm, normal mutated binary cat swarm optimization (NMBCSO), is developed and proposed the multiobjective NMBCSO to thinned antenna array synthesis.

Section 2 presents the brief description of planar array. A detailed description of the proposed MO-NMBCSO is presented in Section 3. The formulation of the problem and a detailed analysis of the results are provided in Section 4. The performance metrics used for quantifying the performance of the algorithms are discussed in Section 5. Lastly Section 6 highlights the achievements of this research work.

\section{Planar Antenna Array}

Consider a planar antenna array of $2 N \times 2 M$ isotropic elements, which is symmetric about the $x$-axis and $y$-axis as

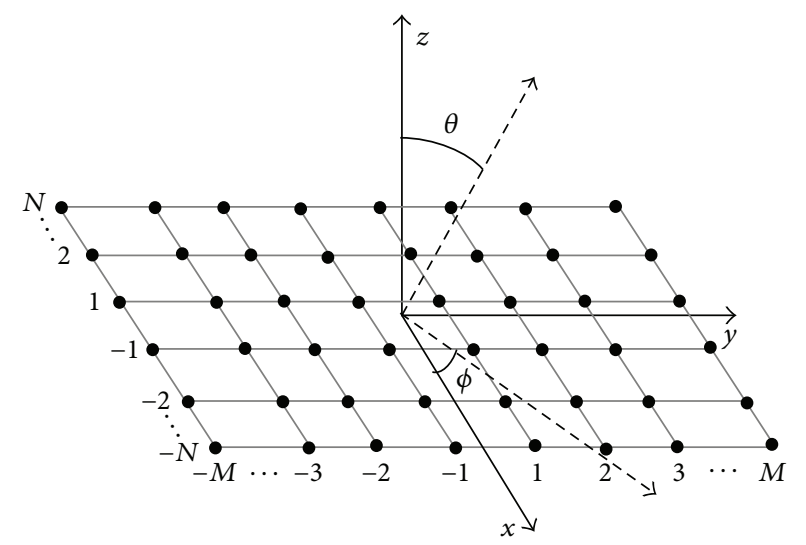

FIGURE 1: Geometry of planar array with uniformly spaced elements.

shown in Figure 1. The array factor for this geometry is given as

$$
\begin{aligned}
\operatorname{AF}(\mathbf{I}, \theta, \phi)= & 4 \sum_{n=1}^{N} \sum_{m=1}^{M} I_{n m} \cdot \cos [\pi \cdot(n-0.5) \cdot U] \\
& \cdot \cos [\pi \cdot(m-0.5) \cdot V]
\end{aligned}
$$

The distance between the elements is assumed to be $0.5 \lambda$, where $U=\sin (\theta) \cos (\phi), V=\sin (\theta) \sin (\phi)$, and $I_{n m}$ represents the amplitude excitation of the $(n, m)$ th element. In thinned arrays, $I_{n m}$ is 1 if the $(n, m)$ th element status is $\mathrm{ON}$ and $I_{n m}$ is 0 if the $(n, m)$ th element status is OFF.

\section{Multiobjective Normal Mutated Binary Cat Swarm Optimization (MO-NMBCSO)}

3.1. Normal Mutated Binary Cat Swarm Optimization (NMBCSO). Cat swarm optimization is modelled by identifying features of a cat's behavior. The features are termed as seeking mode and tracing mode. Cats spend most of the time resting but are always alert by observing the surrounding environment. This behavior is represented in seeking mode. Tracing mode is modelled as behavior of cats while tracing targets. Cats spend a large amount of energy and move very quickly while chasing a target. The above-mentioned modes are structured mathematically to solve different optimization problems.

Normal mutated binary CSO (NMBCSO) is different from the continuous version of CSO. In NMBCSO, the position vector is composed of ones and zeros. This change produces major differences between CSO and BCSO. BCSO is also modelled by identifying features of a cat's behavior as in CSO. In order to deal with binary version, the seeking mode and tracing mode properties are changed while preserving the original behavioral properties of the cat. Multiobjective CSO is proposed in 2012 to solve the real valued multiobjective problems [26]. Pareto ranking [27] scheme is incorporated to discover the nondominated solutions by storing the cats in the external archive. 
3.1.1. Seeking Mode. In seeking mode, the cat is in rest position while being alert. If it wants to move in rest position, the movement is very slow and careful after observing the surrounding environment. Some of the essential factors related to this mode are as follows:

(i) Seeking memory pool (SMP): it indicates how many number of copies of cat to be produced in seeking mode.

(ii) Counts of dimension to change (CDC): it indicates how many number of dimensions to be mutated.

(iii) Mutation probability (MP): the parameter (MP) replaces the parameter of seeking range of selected dimension (SRD) in the traditional real coded CSO. MP indicates the probability of binary mutation. The selected dimensions based on CDC are mutated according to the value of MP.

The steps involved in the seeking mode are as follows:

(1) According to SMP, generate $K$ copies of $i$ th cat.

(2) One copy among $K$ copies maintains the present position and $K-1$ copies undergo mutation. That is, the dimensions of each copy among $K-1$ copies are mutated based on CDC and MP. Each bit in the parent cat will be mutated with a probability of $\mathrm{MP}_{i}^{k}$, which is calculated by the following sigmoid function $[28,29]$ :

$$
\mathrm{MP}_{i}^{k}=\frac{1}{1+e^{-D_{i}^{k}+6}}+\frac{1}{1+e^{D_{i}^{k}+6}},
$$

where $D_{i}^{k}$ is the spread distance generated by the standard normal distributed function $N\left(0, \sigma^{2}\right)$. Normal mutation allows equal likelihood of motion in the positive and negative directions around the cat position. This step allows smaller mutations in the neighborhood of the parent. This allows a more systematic search around the neighborhood of the cat position which enhances the searching capabilities of the binary CSO. The mean and standard deviation is chosen as 0 and 1 , respectively. The sigmoid limiting transformation maps the normal mutation domain into the range of $[0,1]$. After the calculation of probability of each bit in a cat, a uniformly distributed random number " $r$ " in the range $[0,1]$ will be generated to specify the mutation bit through the following equations.

$k$ th bit in $i$ th copy among $K-1$ copies is updated as

$$
\begin{aligned}
y_{i}^{k} & = \begin{cases}1, & \text { if }\left(r<\mathrm{MP}_{i}^{k}\right), \\
0, & \text { else, }\end{cases} \\
x_{i}^{k+1} & =\bmod \left(x_{i}^{k}+y_{i}^{k}, 2\right) .
\end{aligned}
$$

3.1.2. Tracing Mode. In this mode cats trace the targets by changing the positions with their own velocities. The updated velocity equation is given as

$$
V_{i, j}^{g+1}=\omega \cdot V_{i, j}^{g}+C \cdot r \cdot\left(X_{\text {gbest }}-X_{i, j}^{g}\right),
$$

where $i$ is the index of a particle in the swarm, $j$ is the index of position in the particle, $g$ represents the generation number, $V_{i, j}^{g}$ is the velocity of $i$ th particle, $C$ is the acceleration coefficient, $\omega$ is the inertia weight, and $r \in[0,1]$ is the random number. The global $X_{\text {gbest }}$ is selected randomly from the nondominated cat positions from the external archive.

The velocity of $j$ th bit in $i$ th particle is implemented by using sigmoid limiting transformation $S$ :

$$
\begin{aligned}
& S\left(V_{i, j}^{g+1}\right) \\
& = \begin{cases}\frac{1}{1+e^{V_{\max }}} \longrightarrow 0, & V_{i, j}^{g+1}=-V_{\max } \longrightarrow \infty, \\
\frac{1}{2}, & V_{i, j}^{g+1}=0, \\
\frac{1}{1+e^{-V_{\max }}} \longrightarrow 1, & V_{i, j}^{g+1}=V_{\max } \longrightarrow \infty .\end{cases}
\end{aligned}
$$

The sigmoid limiting transformation maps domain of $\left[-V_{\max }, V_{\max }\right]$ into the range of $\left[1 / 1+e^{V_{\max }}, 1 / 1+e^{-V_{\max }}\right]$, which is a subset of $[0,1]$. $j$ th bit in $i$ th particle $X$ is updated as

$$
X_{i, j}^{g+1}= \begin{cases}1, & \text { if } r<S\left(V_{i, j}^{g+1}\right), \\ 0, & \text { if } r \geq S\left(V_{i, j}^{g+1}\right) .\end{cases}
$$

3.2. Multiobjective Approach Using NMBCSO. In order to extend the application of binary CSO algorithm for solving multiobjective problems, the nondominated sorting [30] is incorporated. In nondominated sorting, the individuals are classified according to dominance in ranking scheme. Based on the nondominance, each individual is assigned to rank. The nondominated solutions obtained in the search process are stored in external archive. This idea is carried out to develop MO-NMBCSO.

3.2.1. Algorithm Description of MO-NMBCSO. This algorithm description for the binary valued modified CSO.

(1) Initialize a finite number of cats in $\mathrm{D}$ dimensional solution space randomly and encode it as binary strings.

(2) Initialize the velocity of the cats.

(3) Evaluate the fitness value of each cat and keep the positions of the nondominated solutions in the external archive.

(4) According to MR, cats are moved to seeking mode and tracing mode based on their flags. If the flag of the cat is set to SM, move the cat to the modified SM process; otherwise, apply it to the TM process. In tracing mode, the global best $\left(X_{\text {gbest }}\right)$ is selected randomly form the nondominated cat positions from the archive.

(5) After the completion of two modes, evaluate the fitness value of each cat and update the positions of the nondominated solutions in the archive. 
(6) If the desired solution is achieved, terminate the program; otherwise, the updated cats continue on in the corresponding modes and repeat the steps from 4 to 7 . In seeking mode, the positions of the nondominated solutions based on their ranking are chosen as the updated cats for the next generation. The steps involved in the modified MOCSO algorithm are shown in Figure 2.

\section{Application of MO-NMBCSO to Thinned Planar Antenna Array Synthesis}

In order to achieve the trade-offs between the PSLL and number of active elements and PSLL and FNBW, MONMBCSO and MO-BPSO [11, 31] are applied to synthesize the planar antenna array with the topology as discussed in Section 2. The algorithms are executed for 5 times and the best Pareto front is chosen for all the examples to demonstrate the effectiveness of the proposed approach. The accuracy of the solutions depends on the angular resolution in the azimuth plane. In this case, the radiation pattern of the array is sampled at 451 angles in the azimuth region of $90^{\circ}$ to $180^{\circ}$. The parameters for BCSO are SMP $=5, \mathrm{CDC}=80 \%$, and $\mathrm{MR}=0.8, C=2$, inertia weight is linearly decreased from 0.9 to 0.4 , and $r \in[0,1]$. The parameters for PSO are $C 1$ $=2$ and $C 2=2$, inertia weight is linearly decreased from 0.9 to 0.4 , and $r \in[0,1]$. The archive size is chosen as 100 . The parameters of the algorithms are set through a series of parameter tuning experiments following the guidelines provided in the literature $[24,25]$. The optimization is done with an initial population of 100 arrays for each run. For a fair comparison, the number of function evaluations is set at 50,000 for both algorithms. All the computations are performed using MATLAB on a PC operating at $3 \mathrm{GHz}$ with 2 GB of RAM.

The quality of the nondominated solutions obtained with the MO-NMBCSO and MO-BPSO algorithms is measured by two qualitative metrics $C$ and $I$ and discussed in Section 5.

4.1. Example 1: Trade-Off between PSLL and FNBW. The main motive is to trade off the PSLL in entire $\emptyset$ plane and the number of active elements by searching suitable combination of 1 and 0's. For this purpose, the objective functions are formulated as follows:

(i) Objective function 1: minimization of the normalized PSLL in the entire $\emptyset$ plane is

$$
f_{1}=\max \left(\left|\frac{\mathrm{AF}(\theta, \emptyset)}{\operatorname{AF}(\theta, \emptyset)_{\max }}\right|\right),
$$

where $\operatorname{AF}(\theta, \emptyset)_{\max }$ is the peak of main beam and the fitness $f_{1}$ is valid in the region of $\theta$ and $\emptyset$ excluding the main beam.

(ii) Objective function 2: to minimize the FNBW in entire $\emptyset$ plane,

$$
f_{1}=\max \left(2 \theta_{\mathrm{fn} i}, i=0^{\circ}, 1^{\circ}, \ldots, 90^{\circ}\right),
$$

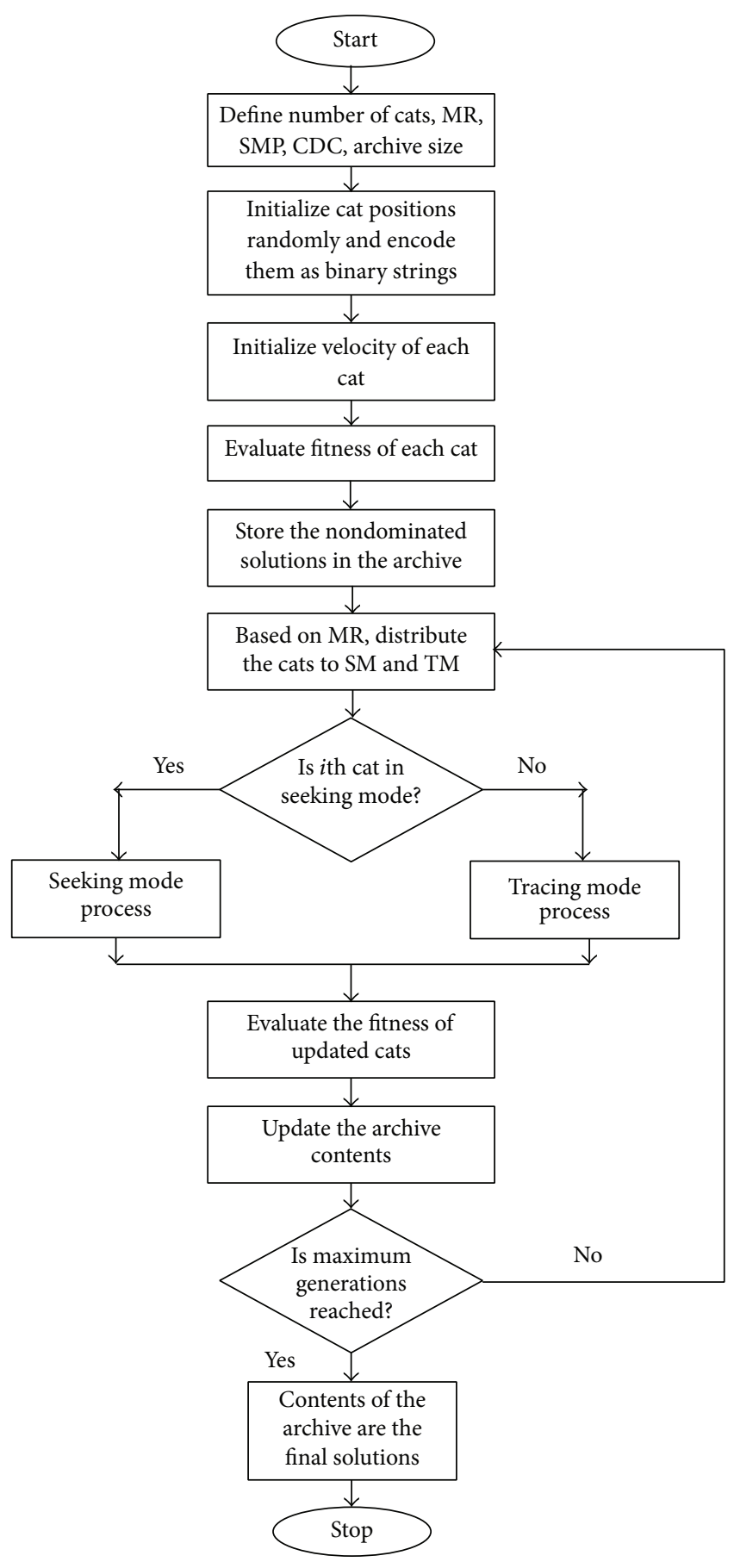

Figure 2: Flow chart for MO-NMBCSO.

where $\theta_{\text {fni }}$ indicates position of first null in $i$ th cutting plane.

Pareto front obtained by trading off the two parameters of PSLL and FNBW is shown in Figure 3. The Pareto fronts over five trails using MO-NMBCSO are shown in Figure 4. For a fair comparison, two Pareto-optimal solutions Array I and Array II obtained using MO-NMBCSO and MO-BPSO which have same FNBW are chosen for comparison as shown 


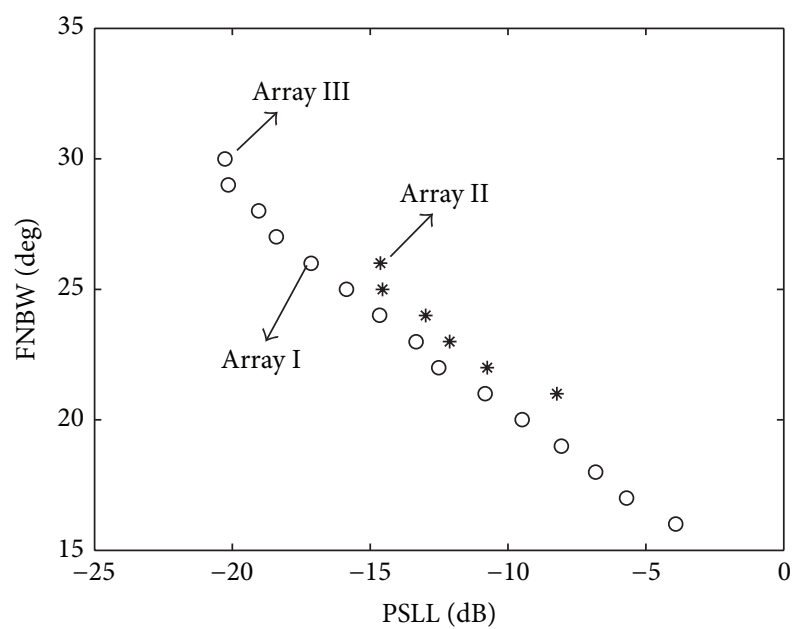

O MO-NMBCSO

* MO-BPSO

FIgURE 3: Pareto fronts for trading off the PSLL and FNBW in entire $\emptyset$ plane using MO-NMBCSO and MO-BPSO.

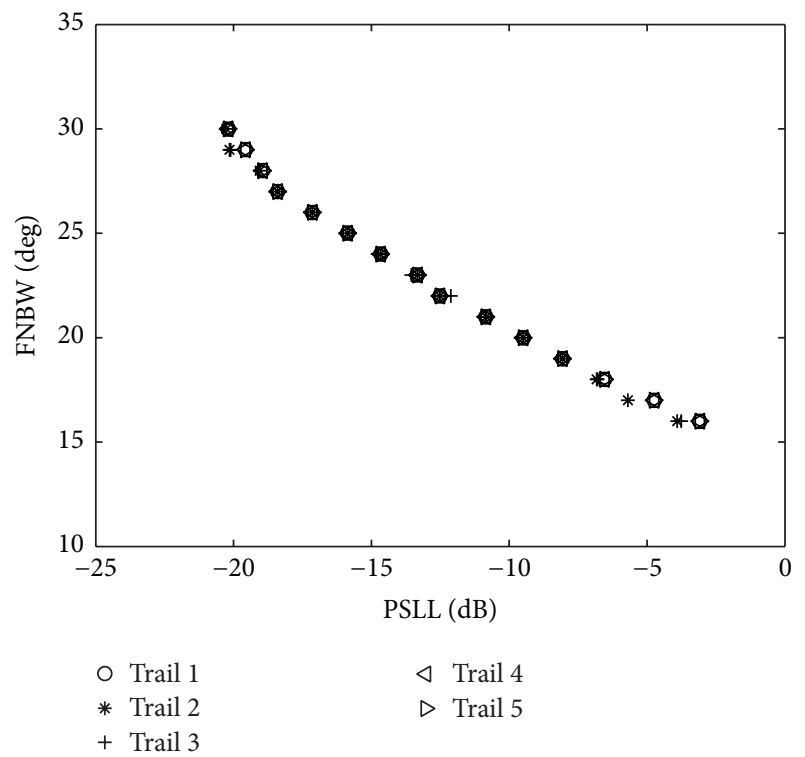

FIgURE 4: Pareto fronts over five trails using proposed MONMBCSO for Example 1.

in Figure 3. This shows that Array I is superior in terms of producing lower PSLL (around $2.5 \mathrm{~dB}$ ) while maintaining similar FNBW as compared to Array II. Also, to show the exploration abilities over the solution landscape, two Paretooptimal solutions Array III and Array II are chosen for comparison which have lowest PSLL obtained using MONMBCSO and MO-BPSO, respectively. This comparison shows that the MO-NMBCSO method proposed in this paper is superior in terms of producing low PSLL $(-20.26 \mathrm{~dB})$ as compared to MO-BPSO $(-14.63 \mathrm{~dB})$. Figure 5 shows the radiation pattern of Array III. The corresponding array element status is also given in Figure 5.

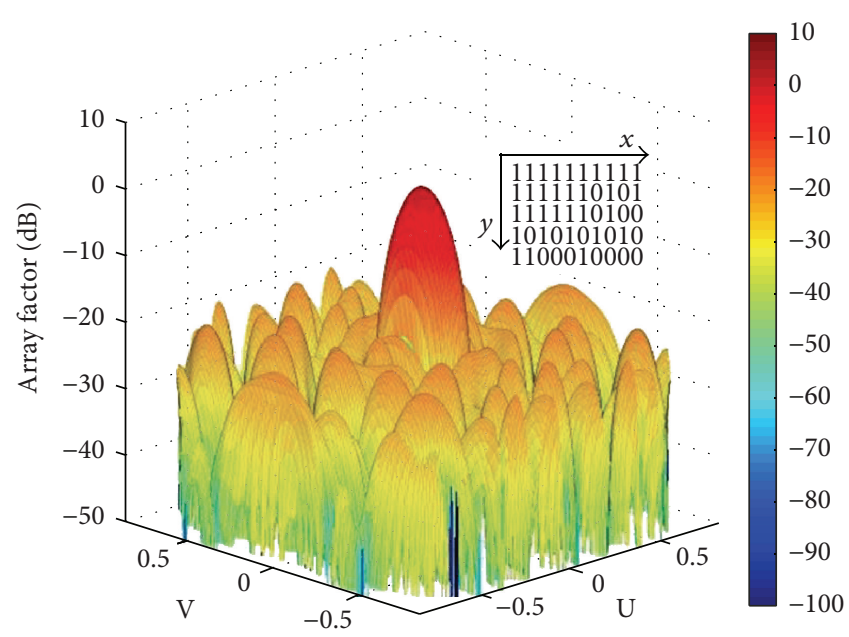

Figure 5: 3D view of the far-field radiation pattern of the MONMBCSO Array III with max PSLL of $-20.19 \mathrm{~dB}$ in entire $\emptyset$ plane.

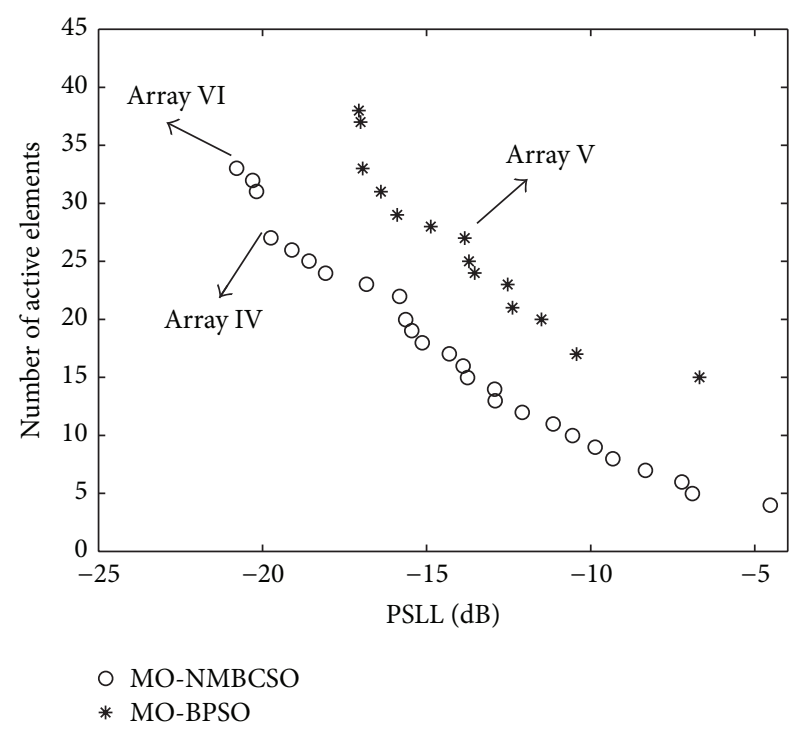

FIGURE 6: Pareto fronts for trading off the PSLL and number of active elements in entire $\emptyset$ plane using MO-NMBCSO and MO-BPSO.

4.2. Example 2: Trade-Offbetween PSLL and Number of Active Elements. The objective functions for trading off the PSLL and FNBW in entire $\emptyset$ plane are given below. The objective function 1 to minimize the normalized the PSLL in the entire $\emptyset$ plane is same as in (7).

Objective function 2: minimization of the number of active elements is

$$
f_{2}=\sum_{i=1}^{N} x_{i}
$$

where $N$ is the number of active elements and $x$ is a set of active elements.

Pareto front obtained by trading off the PSLL and the number of active elements are shown in Figure 6. The Pareto fronts over five trails using MO-NMBCSO are shown in 


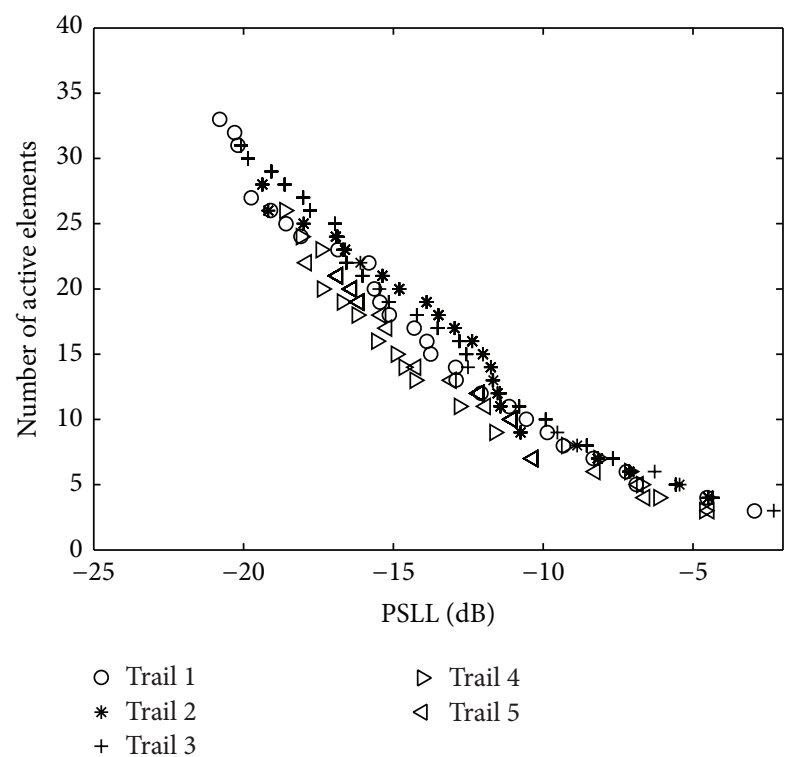

Figure 7: Pareto fronts over five trails using proposed MONMBCSO for Example 2.

Figure 7. Two Pareto-optimal solutions obtained using MONMBCSO and MO-BPSO which have the same 54\% array filling factor are chosen for comparison from Figure 6. These two are referred to as Array IV and Array V, respectively. The PSLL obtained with Array IV is $-19.74 \mathrm{~dB}$, whereas with Array $\mathrm{V}$ it is $-13.84 \mathrm{~dB}$. This shows that MO-NMBCSO is superior in terms of producing lower PSLL (around $6 \mathrm{~dB}$ ) while maintaining same number of active elements as compared to MO-BPSO. The best array in terms of lowest PSLL obtained using MO-NMBSCO is referred to as Array VI. It produces PSLL of $-20.80 \mathrm{~dB}$ with $66 \%$ active elements. The $2 \mathrm{D}$ view of the far-field radiation pattern (U-V cut) of Array VI is shown in Figure 8. Also the far-field radiation patterns at three cutting planes and array element status are shown in Figure 9.

4.3. Comparisons with Published Work. The synthesis of $20 \times$ 10 planar antenna array with low PSLL has been explored previously by using single objective optimization methods $[3,4]$. The optimized array obtained by the proposed novel MO-NMBCSO is compared with previously published work and the comparisons are listed in Table 1. Array VI obtained with MO-NMBCSO offers suppression of PSLL in entire $\emptyset$ plane by around $1.96 \mathrm{~dB}$ and $1.36 \mathrm{~dB}$ as compared to arrays optimized with MGA [3] and OGA [4], respectively. The results demonstrate that the MO-NMBCSO provides an efficient way to control the shape of the radiation pattern by producing low PSLL array designs.

\section{Performance Metrics}

The goals in the multiobjective optimization are to find the solutions as proximate as possible to Pareto-optimal region and to find the solutions as diverse as possible in the Pareto front. Various performance metrics have been proposed to

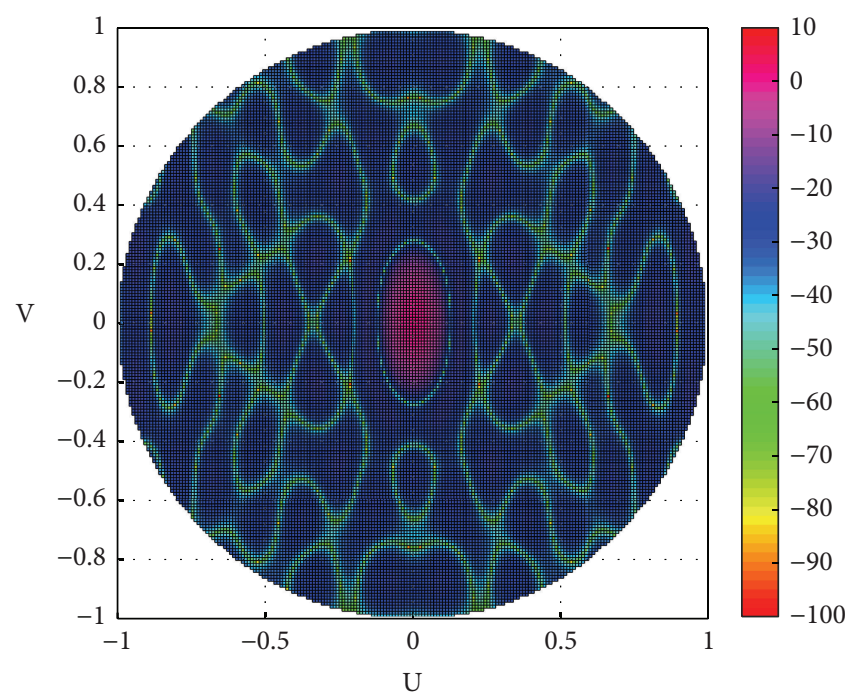

FIGURE 8: 2D-view of the far-field radiation pattern of MONMBCSO Array VI with max PLSS of $-20.80 \mathrm{~dB}$ (U-V cut).

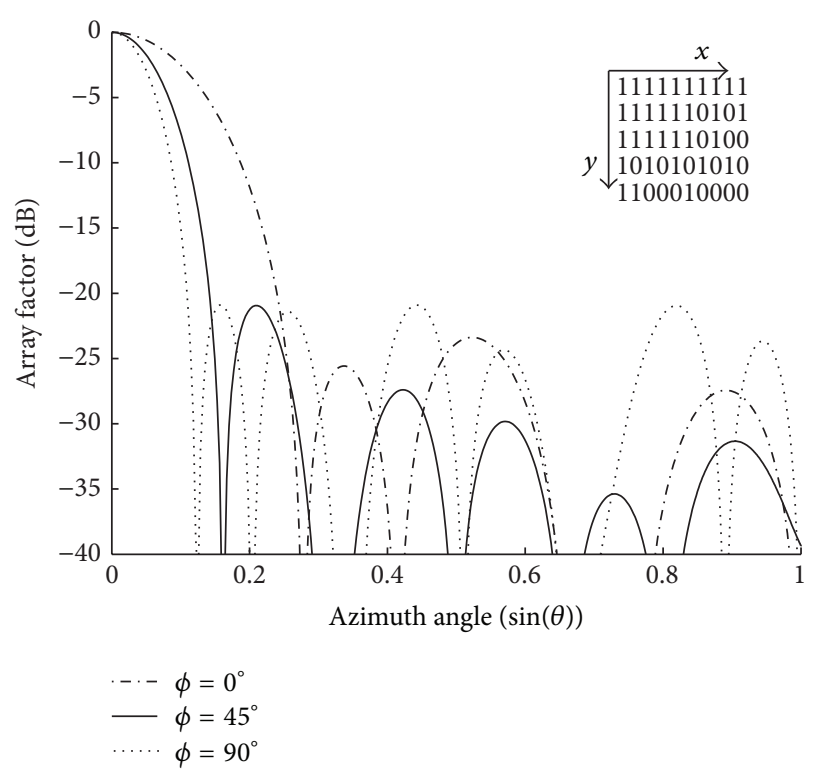

FIGURE 9: Far-field radiation pattern in three cutting planes of Array VI.

measure the goals in multiobjective optimization. Some of the metrics [32, 33] have required the prior knowledge of the true Pareto front, which is unknown in this application. Keeping this limitation in mind, the following metrics are considered in this paper to quantify the performance of various algorithms. Table 2 summarizes the statistical comparison of these metrics for both the algorithms. The best average values are shown in bold font.

5.1. Set Coverage (C-Metric). This metric is proposed by Zitzler and Thiele [32] and it measures the relative spread of the solutions between the two nondominated sets. Let $A$ 
TABLE 1: Comparative results of $20 \times 10$ planar antenna array synthesis.

\begin{tabular}{lccc}
\hline & & & $20 \times 10$ planar antenna array \\
Algorithm & MO-NMBCSO & OGA [4] & Array VI \\
\hline $\begin{array}{l}\text { PSLL in entire } \emptyset \text { plane } \\
\text { (in dB) }\end{array}$ & Array III & -20.80 & -19.44 \\
\hline
\end{tabular}

TABLE 2: Statistical results of different performance metrics for MO-NMBCSO and MO-BPSO.

\begin{tabular}{|c|c|c|c|c|c|}
\hline \multirow{2}{*}{$\begin{array}{l}\text { Performance } \\
\text { metric }\end{array}$} & & \multicolumn{2}{|c|}{ Example 1} & \multicolumn{2}{|c|}{ Example 2} \\
\hline & & MO-NMBCSO & MO-BPSO & MO-NMBCSO & MO-BPSO \\
\hline \multirow{4}{*}{ C } & Best & 1 & 0 & 1 & 0 \\
\hline & Worst & 1 & 0 & 1 & 0 \\
\hline & Mean & 1 & 0 & 1 & 0 \\
\hline & $\mathrm{SD}$ & 0 & 0 & 0 & 0 \\
\hline \multirow{4}{*}{$I$} & Best & 3064.1 & 742.2 & 717.1257 & 47.4662 \\
\hline & Worst & 1440.3 & 625.1 & 588.8575 & 34.32 \\
\hline & Mean & 2216.2 & 702.5 & 666.4571 & 37.5 \\
\hline & $\mathrm{SD}$ & 778.2 & 52.6 & 68.2433 & 6.95 \\
\hline
\end{tabular}

and $B$ be two nondominated sets; the set coverage metric is defined as

$$
C(A, B)=\frac{|\{b \in B \mid \exists a \in A: a \geq b\}|}{|B|},
$$

where $a \geq b$ means that the solution $a \in A$ is weakly dominated by the solution $b \in B$. $|B|$ means the number of solutions in the nondominated set $B$. It measures the amount of solutions in $B$ which are weakly dominated by solutions of $A$. The value $C(A, B)=1$ means that all members in $B$ are weakly dominated by $A$. The value $C(A, B)=0$ represents that none of the solutions in $B$ are weakly dominated by $A$. It has to be noted that both directions have to be considered always, since $C(A, B)$ is not necessarily equal to $1-C(B, A)$.

It can be seen from Table 2 that, for both Examples 1 and 2 , the average value of set coverage metric $(C)$ is 1 and indicates that $100 \%$ of the Pareto-optimal solutions obtained with MO-NMBCSO are weakly dominated by the MO-BPSO solutions.

5.2. Moment of Inertia (I-Metric). It measures the diversity among the nondominated solutions $[32,33]$. It is calculated by measuring the moment of inertia $I$ of the nondominated set. It is defined as

$$
I=\sum_{i=1}^{N} \sum_{j=1}^{Q}\left(f_{i j}-X_{i}\right)^{2},
$$

where $N$ is the number of objectives, $Q$ is the number of solutions in the nondominated set, $f_{i j}$ is $i$ th objective of $j$ th point on the Pareto front, and $X_{i}$ is the centroid for $i$ th objective:

$$
X_{i}=\frac{\sum_{j=1}^{Q} f_{i j}}{Q} .
$$

Higher values of $I$ indicate higher diversity among the nondominated solutions on the Pareto front.

It can be seen from Table 2 that, for both Examples 1 and 2 , the mean value of $I$-metric obtained with $\mathrm{MO}-\mathrm{NMBCSO}$ is more than that of MO-BPSO. It shows that higher diversity among the nondominated solutions on the Pareto front is achieved with MO-NMBCSO compared to MO-BPSO.

\section{Conclusion}

In this communication, a novel multiobjective approach using NMBCSO is proposed for the design of thinned planar antenna arrays. This paper demonstrated several design problems with different planar array configurations. The proposed algorithm is applied to approximate the set of Pareto-optimal solutions by trading off the PSLL and number of active elements and PSLL and FNBW. The results obtained using the MO-NMBCSO and MO-BPSO are compared using two performance metrics, that is, set coverage and moment of inertia. The proposed approach succeeds in achieving better compromised solutions as compared to MO-BPSO method. The obtained optimal designs show that this approach provides an efficient way to control the shape of the radiation pattern with minimum number of active elements. Although this work is focused on the planar thinned antenna array synthesis, the proposed algorithm is also suitable for solving other discrete multiobjective complex problems.

\section{Competing Interests}

The authors declare that they have no competing interests. 


\section{References}

[1] L. Schwartzman, "Element behavior in a thinned array", IEEE Transactions on Antennas and Propagation, vol. 15, no. 4, pp. 571-572, 1967.

[2] R. L. Haupt, “Thinned arrays using genetic algorithms," IEEE Transactions on Antennas and Propagation, vol. 42, no. 7, pp. 993-999, 1994.

[3] K. Chen, X. Yun, Z. He, and C. Han, "Synthesis of sparse planar arrays using modified real genetic algorithm," IEEE Transactions on Antennas and Propagation, vol. 55, no. 4, pp. 1067-1073, 2007.

[4] L. Zhang, Y. C. Jiao, B. Chen, and H. Li, "Orthogonal genetic algorithm for planar thinned array designs," International Journal of Antennas and Propagation, vol. 2012, Article ID 319037, 7 pages, 2012.

[5] G. Oliveri and A. Massa, "Genetic algorithm (GA)-enhanced almost difference set (ADS)-based approach for array thinning," IET Microwaves, Antennas and Propagation, vol. 5, no. 3, pp. 305-315, 2011.

[6] V. Murino, A. Trucco, and C. S. Regazzoni, "Synthesis of unequally spaced arrays by simulated annealing," IEEE Transactions on Signal Processing, vol. 44, no. 1, pp. 119-123, 1996.

[7] W.-B. Wang, Q.-Y. Feng, and D. Liu, "Synthesis of thinned linear and planar antenna arrays using binary pso algorithm," Progress in Electromagnetics Research, vol. 127, pp. 371-387, 2012.

[8] Ó. Quevedo-Teruel and E. Rajo-Iglesias, "Ant colony optimization in thinned array synthesis with minimum sidelobe level," IEEE Antennas and Wireless Propagation Letters, vol. 5, no. 1, pp. 349-352, 2006.

[9] L. Zhang, Y.-C. Jiao, Z.-B. Weng, and F.-S. Zhang, "Design of planar thinned arrays using a Boolean differential evolution algorithm," IET Microwaves, Antennas and Propagation, vol. 4, no. 12 , pp. 2172-2178, 2010.

[10] G. Oliveri, L. Manica, and A. Massa, "ADS-based guidelines for thinned planar arrays," IEEE Transactions on Antennas and Propagation, vol. 58, no. 6, pp. 1935-1948, 2010.

[11] N. Jin and Y. Rahmat-Samii, "Advances in particle swarm optimization for antenna designs: real-number, binary, singleobjective and multiobjective implementations," IEEE Transactions on Antennas and Propagation, vol. 55, no. 3 I, pp. 556-567, 2007.

[12] J. S. Petko and D. H. Werner, "Pareto optimization of thinned planar arrays with elliptical mainbeams and low sidelobe levels," IEEE Transactions on Antennas and Propagation, vol. 59, no. 5, pp. 1748-1751, 2011.

[13] M. A. Panduro, D. H. Covarrubias, C. A. Brizuela, and F. R. Marante, "A multi-objective approach in the linear antenna array design," AEU-International Journal of Electronics and Communications, vol. 59, no. 4, pp. 205-212, 2005.

[14] F. Tokan and F. Güneş, "The multi-objective optimization of non-uniform linear phased arrays using the genetic algorithm," Progress in Electromagnetics Research B, no. 17, pp. 135-151, 2009.

[15] F. Tokan and F. Güne, "Pareto optimal synthesis of the linear array geometry for minimum sidelobe level and null control during beam scanning," International Journal of RF and Microwave Computer-Aided Engineering, vol. 20, no. 5, pp. 557566, 2010.

[16] S. K. Goudos, K. A. Gotsis, K. Siakavara, E. E. Vafiadis, and J. N. Sahalos, "A multi-objective approach to subarrayed linear antenna arrays design based on memetic differential evolution,"
IEEE Transactions on Antennas and Propagation, vol. 61, no. 6, pp. 3042-3052, 2013.

[17] S. Pal, B. Y. Qu, and S. Das, "Optimal synthesis of linear antenna arrays with multi-objective differential evolution," Progress in Electromagnetic Research B, vol. 21, pp. 87-111, 2009.

[18] D. Mandal, A. K. Bhattacharjee, and S. P. Ghoshal, "Comparative optimal designs of non-uniformly excited concentric circular antenna array using evolutionary optimization techniques," in Proceedings of the 2nd International Conference on Emerging Trends in Engineering and Technology (ICETET '09), pp. 619624, Nagpur, India, December 2009.

[19] M. A. Panduro, C. A. Brizuela, J. Garza, S. Hinojosa, and A. Reyna, "A comparison of NSGA-II, DEMO, and EM-MOPSO for the multi-objective design of concentric rings antenna arrays," Journal of Electromagnetic Waves and Applications, vol. 27, no. 9, pp. 1100-1113, 2013.

[20] M. A. Panduro and C. Brizuela, "Evolutionary multi-objective design of non-uniform circular phased arrays," International Journal for Computation and Mathematics in Electrical and Electronic Engineering, vol. 27, no. 2, pp. 549-564, 2008.

[21] C.-Y. Chan and P. M. Goggans, "Multiobjective design of linear antenna arrays using Bayesian inference framework," IEEE Transactions on Antennas and Propagation, vol. 62, no. 11, pp. 5525-5530, 2014.

[22] M. A. Panduro, C. A. Brizuela, and D. H. Covarrubias, "Design of electronically steerable linear arrays with evolutionary algorithms," Applied Soft Computing Journal, vol. 8, no. 1, pp. 46-54, 2008.

[23] S.-C. Chu and P.-W. Tsai, "Computational intelligence based on the behavior of cats," International Journal of Innovative Computing, Information and Control, vol. 3, no. 1, pp. 163-173, 2007.

[24] L. Pappula and D. Ghosh, "Linear antenna array synthesis using cat swarm optimization," AEU-International Journal of Electronics and Communications, vol. 68, no. 6, pp. 540-549, 2014.

[25] G. Panda, P. M. Pradhan, and B. Majhi, "IIR system identification using cat swarm optimization," Expert Systems with Applications, vol. 38, no. 10, pp. 12671-12683, 2011.

[26] P. M. Pradhan and G. Panda, "Solving multiobjective problems using cat swarm optimization," Expert Systems with Applications, vol. 39, no. 3, pp. 2956-2964, 2012.

[27] N. Srinivas and K. Deb, "Muiltiobjective optimization using nondominated sorting in genetic algorithms," Evolutionary Computation, vol. 2, no. 3, pp. 221-248, 1994.

[28] Z. S. W. Y. Lingnan, "Invasive weed optimization algorithm of discrete binary version," Journal of Huazhong University of Science and Technology (Natural Science Edition), vol. 10, pp. 5560, 2011.

[29] H. Wu, C. Liu, and X. Xie, “Thinning of concentric circular antenna arrays using improved binary invasive weed optimization algorithm," Mathematical Problems in Engineering, vol. 2015, Article ID 365280, 8 pages, 2015.

[30] K. Deb, A. Pratap, S. Agarwal, and T. Meyarivan, "A fast and elitist multiobjective genetic algorithm: NSGA-II," IEEE Transactions on Evolutionary Computation, vol. 6, no. 2, pp. 182197, 2002.

[31] C. A. Coello Coello, G. T. Pulido, and M. S. Lechuga, "Handling multiple objectives with particle swarm optimization," IEEE Transactions on Evolutionary Computation, vol. 8, no. 3, pp. 256-279, 2004. 
[32] E. Zitzler and L. Thiele, "Multiobjective evolutionary algorithms: a comparative case study and the strength Pareto approach," IEEE Transactions on Evolutionary Computation, vol. 3, no. 4, pp. 257-271, 1999.

[33] P. M. Pradhan and G. Panda, "Connectivity constrained wireless sensor deployment using multiobjective evolutionary algorithms and fuzzy decision making," Ad Hoc Networks, vol. 10, no. 6, pp. 1134-1145, 2012. 

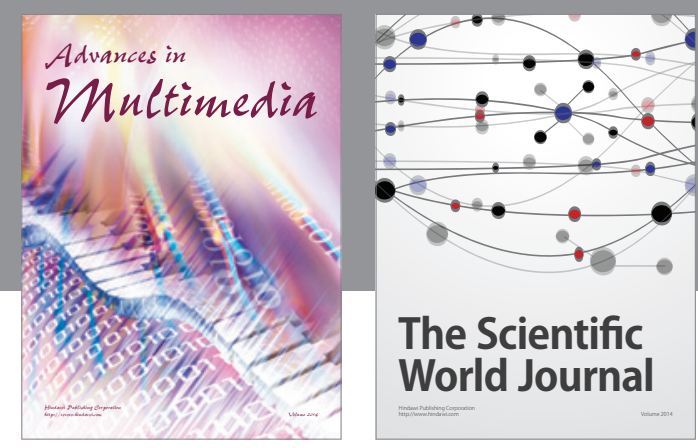

The Scientific World Journal
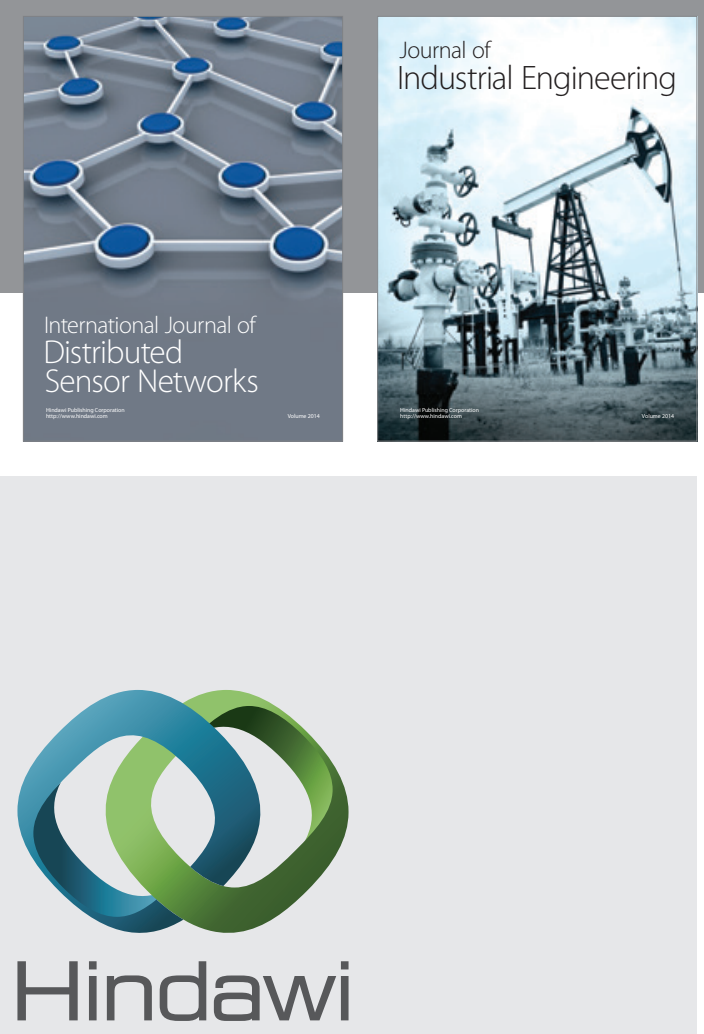

Submit your manuscripts at

http://www.hindawi.com

\section{Computer Networks} and Communications
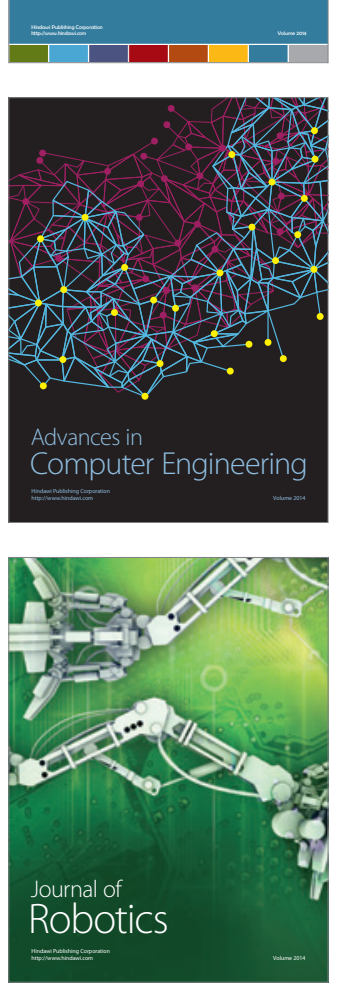
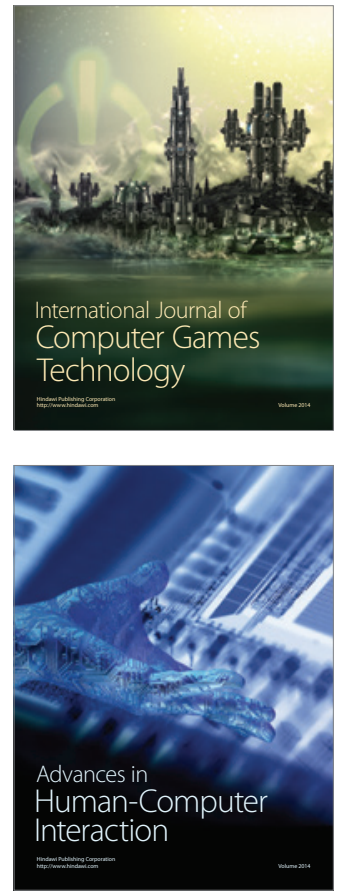
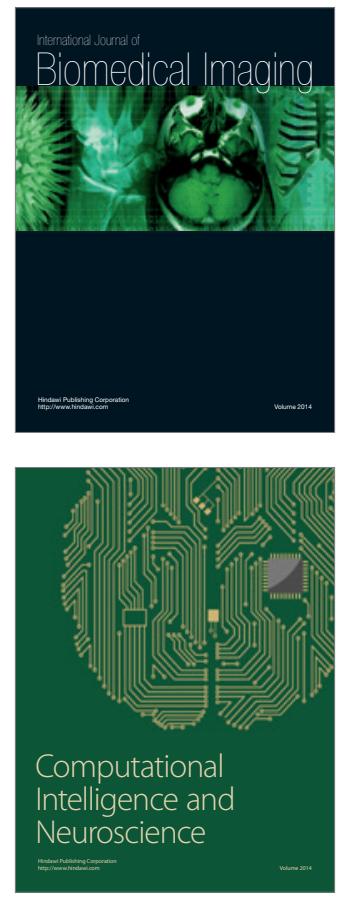
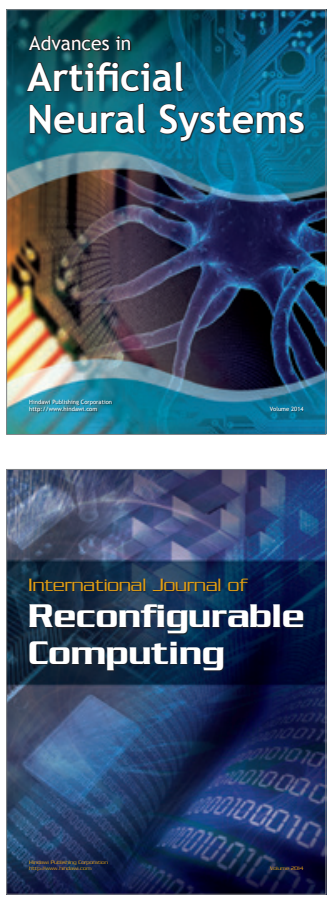
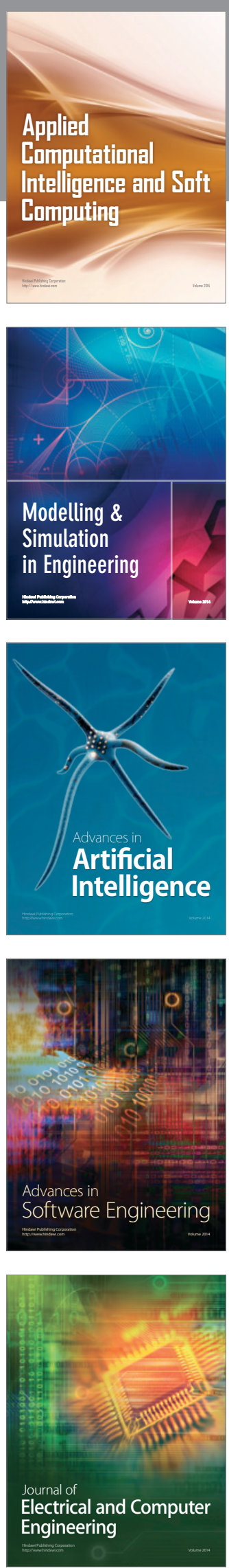